Check for updates

Cite this: Phys. Chem. Chem. Phys., 2020, 22, 18507

Received 6th April 2020

Accepted 20th July 2020

DOI: $10.1039 / \mathrm{d} 0 \mathrm{cp} 01857 \mathrm{k}$

rsc.li/pccp

\title{
Evolution of the structure and dynamics of bovine serum albumin induced by thermal denaturation
}

\author{
Olga Matsarskaia, (D) *a Lena Bühl, ${ }^{\text {ab }}$ Christian Beck, (D) ab Marco Grimaldo, ${ }^{a}$ \\ Ralf Schweins, (D) ${ }^{a}$ Fajun Zhang, (D) ${ }^{b}$ Tilo Seydel, (D) ${ }^{a}$ Frank Schreiber (D) ${ }^{b}$ and \\ Felix Roosen-Runge (iD *cd
}

\begin{abstract}
Protein denaturation in concentrated solutions consists of the unfolding of the native protein structure, and subsequent cross-linking into clusters or gel networks. While the kinetic evolution of structure has been studied for some cases, the underlying microscopic dynamics of proteins has so far been neglected. However, protein dynamics is essential to understand the specific nature of assembly processes, such as diffusion-limited growth, or vitrification of dense liquids. Here, we present a study on thermal denaturation of concentrated solutions of bovine serum albumin (BSA) in $\mathrm{D}_{2} \mathrm{O}$ with and without $\mathrm{NaCl}$. Using small-angle scattering, we provide information on structure before, during and after denaturation. Using quasi-elastic neutron scattering, we monitor in real-time the microscopic dynamics and dynamical confinement throughout the entire denaturation process covering protein unfolding and cross-linking. After denaturation, the protein dynamics is slowed down in salty solutions compared to those in pure water, while the stability and dynamics of the native solution appears unaffected by salt. The approach presented here opens opportunities to link microscopic dynamics to emerging structural properties, with implications for assembly processes in soft and biological matter.
\end{abstract}

\section{Introduction}

The functionality of soft matter and biological systems is often determined by their structure. A case in point are proteins, the biological functions of which depend on a combination of both structural features and dynamics. As the result of an elaborate and intriguing folding process in living cells, protein structure is determined by a subtle interplay of hydration-driven and electrostatic interactions and is known to be highly dependent on external parameters such as $\mathrm{pH}$, ionic strength, solvent composition, pressure and temperature as well as the concentration of the proteins themselves (crowding) and genetic mutations. ${ }^{1,2}$ In fact, extreme conditions induced by (combinations of) these parameters can lead to drastic changes in protein structure including denaturation, unfolding and aggregation. In a medical context, the latter processes have been linked to several serious human pathologies, such as Alzheimer's disease,$^{3}$ inflammation-related conditions ${ }^{4}$ or eye cataract. ${ }^{5}$ Interestingly, certain extremophilic

\footnotetext{
${ }^{a}$ Institut Laue-Langevin, 71 Avenue des Martyrs, 38042 Grenoble, France. E-mail: matsarskaia@ill.eu; Tel: +33 (0)4 57428256

${ }^{b}$ Institut für Angewandte Physik, Auf der Morgenstelle 10, 72076 Tübingen, Germany ${ }^{c}$ Department of Biomedical Sciences and Biofilms-Research Center for Biointerfaces (BRCB), Faculty of Health and Society, Malmö University, Sweden.

E-mail: felix.roosen-runge@mau.se; Tel: +46 (0)40 6657969

${ }^{d}$ Division of Physical Chemistry, Lund University, Naturvetarvägen 14, 22100 Lund, Sweden
}

organisms such as some types of bacteria, are able to survive and even thrive under drastic conditions such as high pressure in the deep sea, ${ }^{6}$ high ionic strength ${ }^{7,8}$ or strongly elevated temperatures in hot springs. ${ }^{9}$ In addition to aspects such as the presence of osmolytes preventing unfolding, ${ }^{10}$ the abilities of such microorganisms to resist these extremes can be traced back to specific adaptations of some types of proteins ${ }^{11-15}$ which evolved to prevent unfolding and a concurrent loss of functionality.

While protein denaturation and unfolding are mostly undesirable in the context of living cells and elaborate mechanisms have evolved in order to avoid these processes, controlled denaturation of proteins is an important step in the food industry where it allows for the formation of protein particles. The latter can play crucial roles in the structuring of food products. ${ }^{16}$

Given their high complexity and ubiquitous presence in nature and everyday life, studying the effects of thermal denaturing on proteins can thus provide valuable information on protein folding as well as on the relation between their structure and function. This study intends to elucidate both the structure and dynamics during protein denaturation by investigating the thermal denaturation of aqueous solutions of bovine serum albumin (BSA) with varying ionic strengths. i.e. without added salt and with $150 \mathrm{mM} \mathrm{NaCl}$. The reason for choosing $\mathrm{NaCl}$ for our studies is its relevance in the physiological context. Amongst its other functions such as maintaining the membrane potential and transducing signals, ${ }^{1} \mathrm{NaCl}$ mediates the ionic 
strength in blood, ${ }^{17,18}$ of which serum albumins are also an essential constituent, thus motivating their investigations in the presence of $\mathrm{NaCl}$.

Before elaborating on the details of the present study, we will provide a short summary of a selection of previous studies concerned with the thermal denaturation of BSA, in particular in the presence of additives such as salt.

The denaturation temperature of BSA under various conditions has been subject to several studies. Using differential scanning calorimetry (DSC), Giancola et al. ${ }^{19}$ determined the denaturation temperature of $20 \mathrm{mg} \mathrm{ml}^{-1}$ BSA in $10 \mathrm{mM}$ phosphate buffer and with $150 \mathrm{mM} \mathrm{NaCl}$ to be $337 \mathrm{~K}\left(64{ }^{\circ} \mathrm{C}\right)$ at $\mathrm{pH}=7$. Another DSC study by Yamasaki and Yano ${ }^{20}$ reports an onset of denaturation of $2 \%$ BSA solutions with an ionic strength of $0.1 \mathrm{M} \mathrm{NaCl}$ at $51.9{ }^{\circ} \mathrm{C}$ and the temperature of denaturation itself at $64.3^{\circ} \mathrm{C}$. In addition, a FTIR study by Murayama and Tomida ${ }^{21}$ determined that in a solution of $20 \mathrm{mg} \mathrm{ml}^{-1} \mathrm{BSA}$ in $\mathrm{D}_{2} \mathrm{O}$ with $100 \mathrm{mM} \mathrm{NaCl}$, irreversible changes occur after $76{ }^{\circ} \mathrm{C}$. For a BSA solution of $500 \mathrm{mg} \mathrm{ml}^{-1}$ in $\mathrm{D}_{2} \mathrm{O}$, Hennig et al. ${ }^{22}$ report a denaturation process taking place in the temperature range between 60 and $81{ }^{\circ} \mathrm{C}$. These studies show that the exact denaturation temperature determined depends on the experimental conditions and methods. In this paper, we provide the denaturation temperature values determined from our quasielastic neutron scattering data in Table 1.

Using differential scanning calorimetry (DSC) and Fouriertransform infrared spectroscopy (FTIR), Boye et al. ${ }^{23}$ performed a detailed investigation of the effect of $\mathrm{pH}$, sugars, reducing and denaturation agents, detergents and $\mathrm{NaCl}$ on the temperatureinduced gelation of BSA. In the presence of all additives studied and at $\mathrm{pH}$ values between 3 and 9 , the authors found a decrease of the $\alpha$-helix content and an increase in $\beta$-sheets and random coils. In addition, they report an enhanced stability of BSA with respect to denaturing in the presence of $0.5 \mathrm{M} \mathrm{NaCl}$, reflected in an increased denaturation temperature compared to BSA samples without added $\mathrm{NaCl}$. These results are corroborated by a calorimetric study by Giancola et al. ${ }^{19}$ and by rheological studies of heat-induced gelation of BSA by Tobitani and Ross-Murphy. ${ }^{24,25}$ These authors report a strong sensitivity of the gelation time of low-concentration BSA solutions to the presence of $\mathrm{NaCl}$. Moreover, an increase of the gelation temperature with decreasing BSA concentration was observed. The structure formed by thermally denatured BSA solutions was studied by Lefebvre et $a .^{26}$ via rheology and small-angle neutron scattering. Their results revealed the presence of compact 3D clusters with a smooth surface. On the level of the individual BSA molecules constituting these clusters, a $20 \%$ increase of their Stokes radii was found upon denaturation. ${ }^{26}$

At high $\mathrm{NaCl}$ concentrations ( $2 \mathrm{M}$ ), Boye et al. observed more pronounced aggregation upon heating. ${ }^{23}$ A detailed DSC study on the interplay of denaturation, aggregation and gelation was performed by Barone et al., ${ }^{27}$ comparing the behaviors of BSA and its human analog, human serum albumin (HSA). Both proteins showed maxima in their denaturation temperatures at a $\mathrm{pH}$ of 5.5, the absolute values of the denaturation enthalpy being lower for HSA. At low pH, denaturation and aggregation coincided. ${ }^{27}$ Furthermore, Vetri et al. ${ }^{28}$ performed fluorescence studies on the aggregation behavior of BSA and HSA. In spite of the strong similarities of these two proteins, their studies revealed different pathways towards aggregation for these two proteins. Moreover, their results underlined the significance of tertiary structure changes in BSA aggregation. As a general finding obtained using fluorescence, FTIR and light scattering, Militello et al. ${ }^{29,30}$ demonstrated the important role of the $\mathrm{pH}^{-}$ mediated net protein charge in the aggregation behavior higher net charges lead to formation of small, $\beta$-sheet-type aggregates, whereas lower net charges imply the formation of more polydisperse aggregates. ${ }^{29}$ Circular dichroism and fluorescence investigations by Bhattacharya et al. ${ }^{31}$ showed that, at low $\mathrm{pH}$ and high temperatures, molten-globule-like BSA oligomers assembled into amyloid-like fibrils. It is important to note that $\mathrm{pH}$ changes can per se induce conformational changes in BSA. ${ }^{32}$ This can be counteracted by additives, as has been shown in a recent study combining small-angle X-ray scattering, spectroscopy, UV and fluorescence by Del Giudice et al.,$^{33}$ demonstrating that the binding of palmitic acid can stabilize the native form of HSA even at $\mathrm{pH}$ values which would typically induce unfolding. The co-binding of ibuprofen and propofol was furthermore shown to prevent HSA unfolding in the presence of urea. ${ }^{34}$

Different studies using quasi-elastic neutron scattering report on the dynamics of denatured BSA. ${ }^{35}$ Using neutron timeof-flight spectroscopy, strongly heterogeneous slow dynamics was found in BSA after chemical denaturation by guanidinium hydrochloride. ${ }^{36}$ In the same system of BSA with guanidinium hydrochloride, Ameseder et al. observed a high internal friction consistent with predictions from polymer theory. ${ }^{37}$ Grimaldo et al. ${ }^{38}$ proposed a picture of hierarchical dynamics in the thermally denatured state of BSA, where global translational diffusion is arrested, while localized internal dynamics becomes enhanced. Interestingly, while global dynamics was irreversibly arrested, the localized dynamics returned to its native state. ${ }^{38}$ Finally, Hennig et al. presented a showcase of following the process of thermal denaturation of BSA in solution, observing a dynamical slowing down upon thermal denaturation, as expected intuitively. ${ }^{22}$

Given the complex denaturation behavior tuned by different environmental parameters, we limit our study to a more simple basic question, namely, how the variation of the ionic strength as a sole parameter affects the denaturation process. Focusing on this question, we report on a comprehensive characterization of both static and dynamic aspects of thermal denaturation of BSA by a combination of small-angle, elastic and inelastic neutron scattering methods. We explore crowded BSA solutions with concentrations from 150 to $500 \mathrm{mg} \mathrm{ml}^{-1}$ in the presence and absence of NaCl. First, we use small-angle neutron scattering (SANS) to characterize time-averaged static features including protein interactions in the native solution, and the characteristic length and fractal dimensions of our systems. Second, information on diffusive dynamics of BSA in the process of thermal denaturation is obtained by recording quasi-elastic neutron scattering (QENS) by means of neutron backscattering (NBS). QENS is a sensitive tool to monitor changes in neutron energy during the scattering process in 
the sample, and thereby provides information on the underlying dynamics on pico- to nanosecond time scales. Here, we focus on so-called elastic and inelastic fixed window scans (EFWS/IFWS). From this technique, we obtain information on the temperaturedependent apparent mean-squared displacement, $\left\langle u^{2}\right\rangle$, of BSA, reflecting the dynamical confinement, as well as a direct measure of diffusivity. Our approach thus provides us with a framework for a detailed and comprehensive characterization of both static and dynamic aspects of protein denaturation as a function of protein concentration, temperature and ionic strength.

This paper is organized as follows. In Section 2, we describe the sample preparation and the principles of the experimental methods (small-angle neutron scattering, quasi-elastic neutron backscattering) employed in this study. In Section 3, we discuss the results obtained before providing conclusions on the work presented here in Section 4.

\section{Materials and methods}

\subsection{Sample preparation}

BSA, $\mathrm{D}_{2} \mathrm{O}$ (99.99\% atom purity) and $\mathrm{NaCl}$ were purchased from Sigma Aldrich (now Merck KgaA, Darmstadt, Germany). Prior to sample preparation, BSA powder was dried over silica beads in order to reduce incoherent scattering due to potential contamination by water $\left(\mathrm{H}_{2} \mathrm{O}\right)$ absorbed from the surrounding air. $\mathrm{D}_{2} \mathrm{O}$ was degassed under vacuum to minimize shifts in $\mathrm{pD}$ and potential pD-related protein structural changes.

SANS and QENS samples containing the desired BSA and $\mathrm{NaCl}$ concentrations were prepared by mixing the required amounts of protein in powder form and concentrated $\mathrm{NaCl}$ stock solutions. The respective neutron scattering experiments were then performed as described in Sections 2.2 and 2.3.

\subsection{Small-angle neutron scattering (SANS)}

Solution small-angle neutron scattering (SANS) probes the shape, the spatial distribution and the interactions between solute particles by detecting the radiation scattered by the sample as a function of momentum transfer $q$. The differential scattering cross-section of a SANS experiment can be expressed as ${ }^{39,40}$

$$
\frac{\mathrm{d} \sigma}{\mathrm{d} \Omega}(q)=n(\Delta \rho)^{2} V_{\text {part }^{2}} P(q) S(q)
$$

where $n$ is the particle number density, $\Delta \rho$ is the difference in scattering contrast between the solvent and the particles, and $V_{\text {part }}$ is the volume of a single particle. ${ }^{39,40}$ The term $P(q)$ is referred to as the particle form factor, determined by the protein conformation. ${ }^{40,41}$ The structure factor, $S(q)$ (for details, see e.g. ref. 42-44), characterizes the correlations and thus the interaction between proteins in solution, and the position of the principal peak $q^{*}$ can be related to a characteristic distance $\xi=2 \pi / q^{*}$ in the sample.

For cross-linked network structures, we use the relation $I(q) \sim q^{-D}$ in the low- $q$ region, where the fractal dimension $D$ of the system provides additional structural information on the system in question. ${ }^{44-46}$

The SANS experiments described here were performed on the small-angle scattering instrument D11 (ILL, Grenoble, France). ${ }^{47}$
Approximately $750 \mu \mathrm{l}$ of sample solution were filled into square quartz cells (Hellma, Müllheim, Germany) with a path length of $2 \mathrm{~mm}$ and the cells were placed on a temperature-controlled sample holder. In order to obtain insights into the structural properties of the protein solution during denaturation, we collected scattering profiles at defined temperatures reaching from room temperature to above the denaturation temperature. Furthermore, we collected a final scattering profile for the denatured sample after cooling it back to room temperature.

Every sample was measured at sample-to-detector distances of 1.5 and $8 \mathrm{~m}$ to maximize the range of accessible length scales providing structural information. The incoming neutron wavelength, $\lambda$, was $6 \AA$ with a full width-half maximum (FWHM) wavelength spread of $9 \%$. A $q$ range from 0.0064 to $0.4247 \AA^{-1}$ was covered. Scattered neutrons were detected via a ${ }^{3} \mathrm{He}$ gas detector (CERCA) with a pixel size of $3.75 \times 3.75 \mathrm{~mm}^{2}$ and a total pixel number of $256 \times 256$. Data were calibrated to an absolute scale using water $\left(\mathrm{H}_{2} \mathrm{O}\right)$ scattering intensity, $\mathrm{d} \Sigma / \mathrm{d} \Omega=$ $0.983 \mathrm{~cm}^{-1}$ as a secondary calibration standard. Raw data were saved in the Nexus (.nxs) format. ${ }^{48}$ Prior to further analysis, all scattering profiles obtained were corrected for both transmission and background scattering.

\subsection{Elastic and inelastic fixed window scans (E/IFWS) of quasi-elastic neutron scattering (QENS)}

Quasi-Elastic Neutron Scattering (QENS) encompasses several scattering techniques that allow to measure both the momentum and energy change of neutrons that is induced by the scattering event in the sample. As the central experimental quantity, the dynamic scattering factor $S(q, \omega)$ is obtained, where $q$ is the structure vector related to the momentum change, and $\hbar \omega$ is the energy change of the neutron in the sample. ${ }^{35}$

Importantly, choosing deuterated solvents, the incoherent neutron scattering from the hydrogen of the protein molecules in the sample dominates the signal, and solvent contributions can be modeled consistently. As hydrogen is rather uniformly distributed in proteins, this experimental protocol provides a unique tool to investigate the averaged dynamics of proteins on (sub)nanosecond time and Ångstrom length scales. On these length and time scales, translational self-diffusion and rotational diffusion are convoluted with internal dynamics. ${ }^{35}$

In the present study, neutron backscattering (NBS) at the spectrometer IN16B (ILL, Grenoble) was used to measure the QENS signal with a high energy resolution $\Delta(\hbar \omega) \approx 0.8 \mu \mathrm{eV}$ FWHM while accessing momentum transfers in the range $q \approx$ 0.2-2 $\AA^{-1}$. The samples $(1.2 \mathrm{ml}$ each) were kept in indiumsealed double-walled cylindrical aluminum cans with a gap of $\Delta r=0.15 \mathrm{~mm}$ holding the liquid sample solutions. The temperature was controlled using a standard Orange cryofurnace.

In the conventional mode, NBS allows to record full QENS spectra $S(q, \omega)$ from a sample within an energy range of typically $-30 \mu \mathrm{eV} \leq \hbar \omega \leq+30 \mu \mathrm{eV}$, requiring at least 2-4 hours of acquisition time per spectrum. Typically, these data are then fitted with model functions, and provide information, e.g., on hierarchical protein dynamics at few selected temperatures during denaturation. ${ }^{38}$ 
So-called fixed window scans (FWS) measure the intensity for only a fixed energy transfer $\omega_{0}$, i.e. either elastic (EFWS, $\omega_{0}=0$ ) or inelastic (IFWS, $\omega_{0} \neq 0$ ) scattering. ${ }^{49}$ Importantly, this approach allows to focus on the rather high scattering intensity at small energy transfers, which reduces the counting time per point to below one minute. The dramatically reduced counting time per point for E/IFWS compared to full spectra enables both parameter scans, and time-resolved monitoring of temporal dynamical evolution. While this study mixes these approaches by real-time monitoring the dynamical evolution driven by a temperature scan, EFWS and IFWS are particularly promising for future studies focusing, e.g., purely on the evolution of dynamics during assembly and nucleation. ${ }^{50,51}$

The conventional analysis of EFWS is based on the Gaussian approximation valid in the limit of low $q$ and yielding an apparent mean squared displacement $\left\langle u^{2}\right\rangle,{ }^{52}$ which characterizes the dynamical confinement of motions within the resolution time of the instrument (for IN16B: $\sim 10 \mathrm{~ns}$ ). Different extensions have been suggested $^{53-55}$ to introduce effects of dynamical heterogeneity, and allow data fitting beyond the immediate low $q$ limit, mainly targeted at hydrated protein powders. We employ the polynomial approach ${ }^{22}$

$$
\ln (S(q, \omega=0))=-\frac{1}{3}\left(b+\left\langle u^{2}\right\rangle q^{2}+c q^{4}\right)
$$

which includes a formal second order term of the cumulant expansion, and has been proven to provide reliable fit results for proteins in solution. Here, $b, c$ and $\left\langle u^{2}\right\rangle$ are scalar fit parameters. For the extraction of $\left\langle u^{2}\right\rangle$, we subtracted the scattering of the empty can using a full Paalman-Pings absorption correction. We used a $q$ range between $0.44-1.1 \AA^{-1}$ for the fitting. We remark that the coherent scattering intensity is around $2 \%$ of the total scattering even for the lowest fitted $q=0.44 \AA^{-1}$, which supports the notion that the data can be analyzed as pure incoherent scattering. We show an exemplary data set for the determination of $\left\langle u^{2}\right\rangle$ in Fig. 1. The data

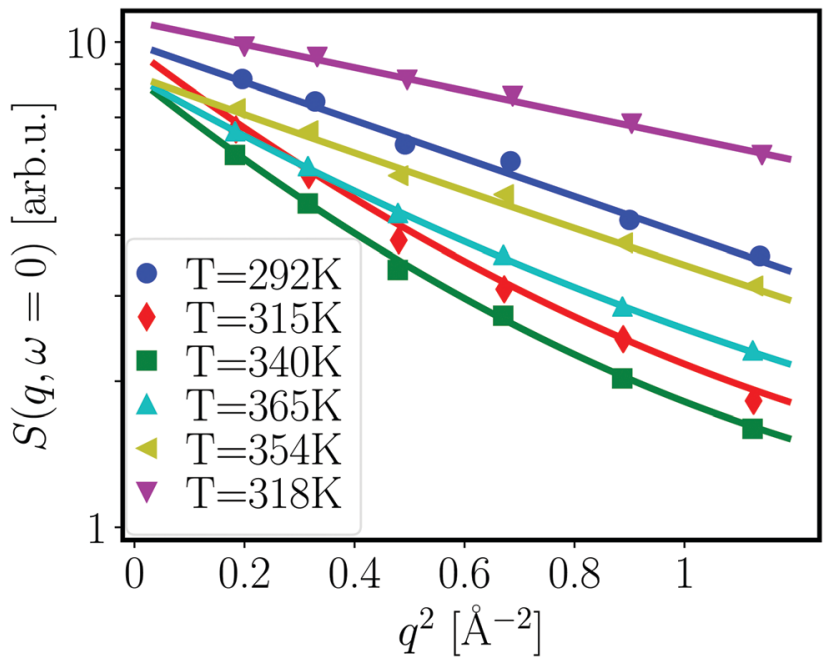

Fig. 1 Normalized $q$-dependent elastic scattering intensities (symbols, see text) for different temperatures (see legend), and fits (lines) for $500 \mathrm{mg} \mathrm{ml}^{-1}$ BSA without $\mathrm{NaCl}$. Note that the data for $T=354 \mathrm{~K}$ and $318 \mathrm{~K}$ were recorded during cooling, the other data during heating. Error bars are smaller than the symbol sizes.
(500 $\mathrm{mg} \mathrm{ml} \mathrm{m}^{-1} \mathrm{BSA}$ without $\mathrm{NaCl}$ ) can be well described by the polynomial fits applied ( $c f$. eqn (2); solid lines in Fig. 1). Obviously, when changing temperature, the profile switches from a rather linear relation $(292 \mathrm{~K})$ to more curved profiles (315-365 K), until it eventually recovers a linear relation after denaturation and cooling down (318 K). The polynomial fit function thus allows to extract $\left\langle u^{2}\right\rangle$ in a systematic way from more complex curved profiles as well as from linear profiles for which the Gaussian approximation would also work.

A standard analysis of IFWS has not yet been established. Potential approaches include semi-quantitative analysis based on the pure IFWS intensity ${ }^{49}$ and the generalized mean-squared displacement. ${ }^{51}$ Here, we use a new approach exploiting the ratio between the intensity of IFWS and EFWS,

$$
A(q, \omega)=\frac{S(q, \omega)}{S(q, \omega=0)} .
$$

The absolute value of $A(q, \omega)$ is related to the dynamics: larger ratios signify a broader $S(q, \omega)$, which implies faster dynamics. Here, we focus only on the relative change with temperature change. We remark that a direct quantitative extraction of dynamical parameters such as diffusion coefficients might be possible if the spectral line shape is well-known, e.g., for simple model systems.

For the ratio analysis, we subtracted the empty can and solvent signal using EFWS and IFWS scans of pure $\mathrm{D}_{2} \mathrm{O}$. We stress that for the ratio analysis, residual coherent contributions would only have an effect, if collective and self-dynamics were strongly different, which is unlikely as no correlation peaks are observed at the small length scales corresponding to the $q$ range from $0.2-2 \AA^{-1}$.

In this study, we combine EFWS and IFWS at $\omega=1.3 \mu \mathrm{eV}$ (cf. eqn (3)) during heating and cooling ramps in concentrated protein solutions, i.e. we monitor the dynamical evolution in real time while continuously changing temperature as a control parameter. We used counting times of 25 and $40 \mathrm{~s}$ for EFWS and IFWS, respectively, alternating between the energy offsets for the full duration of the experiment. The heating and cooling ramp rates were set to $0.21 \mathrm{~K} \mathrm{~min}^{-1}(295 \mathrm{~K} \rightarrow 370 \mathrm{~K}), 0.325 \mathrm{~K} \mathrm{~min}^{-1}$ $(295 \mathrm{~K} \rightarrow 325 \mathrm{~K})$, and $1 \mathrm{~K} \mathrm{~min}^{-1}(325 \mathrm{~K} \rightarrow 295 \mathrm{~K})$, respectively. We remark that the cooling ramp of $150 \mathrm{mg} \mathrm{ml}^{-1}$ BSA with $150 \mathrm{mM}$ $\mathrm{NaCl}$ had a programming error, which resulted in an overshooting temperature, and faster cooling back.

For the interpretation of the data obtained, we focus on relative changes in the diffusivity of samples as a function of different conditions, i.e., protein and salt concentration, and use the combination of EFWS and IFWS as a novel indicator for timeresolved changes of dynamical evolution during a temperature scan.

\section{Results and discussion}

\subsection{SANS: structural changes upon thermal denaturation}

The evolution of the structural properties of the BSA-NaCl samples upon denaturation was probed by SANS. The temperaturedependent SANS data are presented in Fig. 2. 

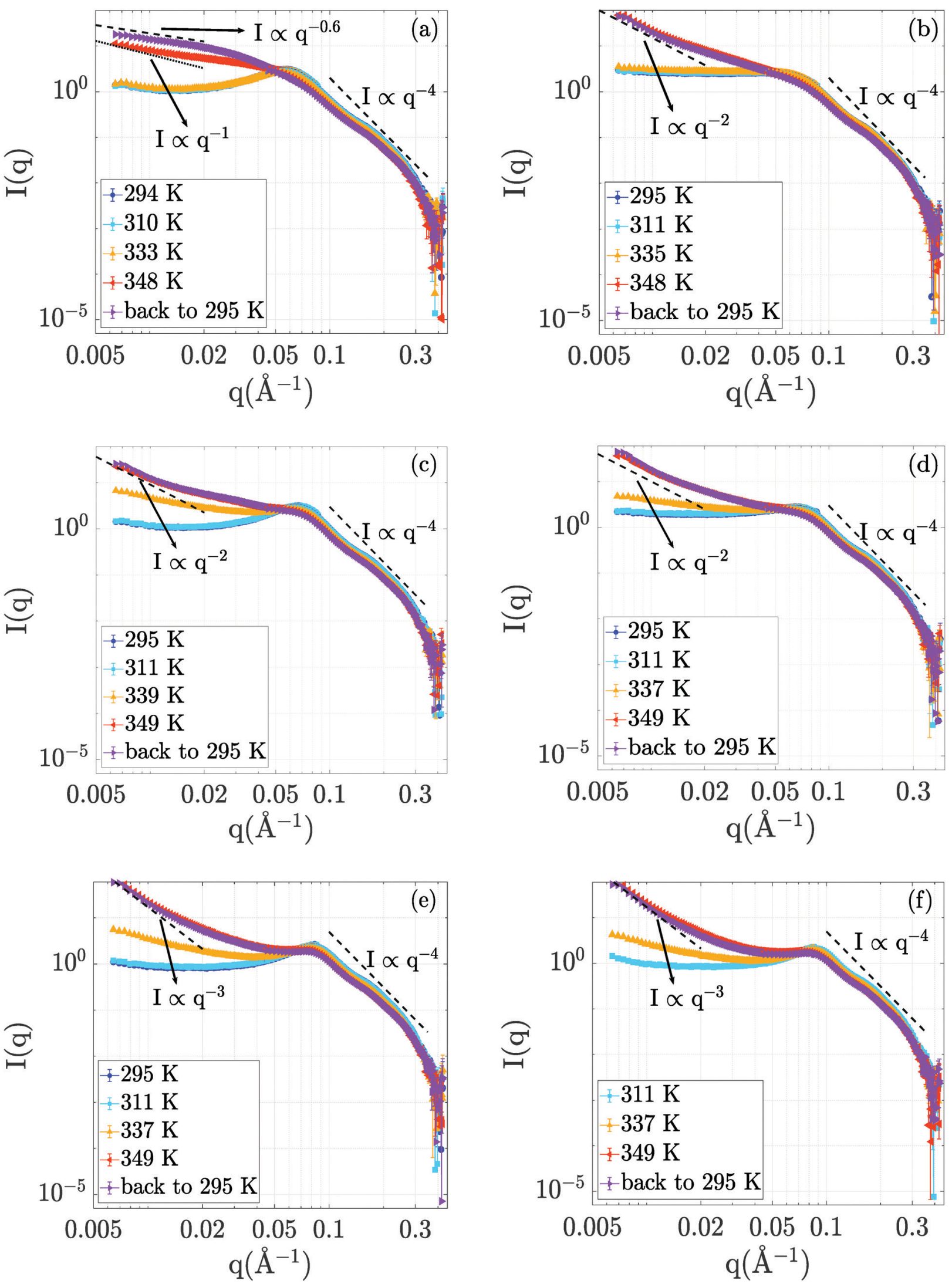

Fig. 2 SANS profiles of samples with different BSA concentrations with and without $\mathrm{NaCl}$ ((a), (c) and (e): 150,250 and $500 \mathrm{mg} \mathrm{ml}^{-1} \mathrm{BSA}$ without $\mathrm{NaCl}$; (b), (d) and (f): 150,250 and $500 \mathrm{mg} \mathrm{ml}^{-1} \mathrm{BSA}$ with $150 \mathrm{mM} \mathrm{NaCl}$ ). All samples were prepared at RT ( $295 \mathrm{~K}$ ), heated to $350 \mathrm{~K}$ and then cooled back to $295 \mathrm{~K}$ to assess the effects of thermal denaturation on the sample properties. Low-q slopes of the samples are given by the dashed lines shown in the respective subplots to allow visual comparison of the different low $q$ scaling. In the high- $q$ region, the Porod relation of $/(q) \propto q^{-4}$ is indicated. 
Starting from the data at room temperature (295 K), we obtain a picture of protein interactions tuned by ionic strength and concentration, consistent with earlier findings. ${ }^{56}$ The electrostatic repulsion leads to a correlation peak in the structure factor $S(q)$, such as the clearly visible peak at $q_{\text {corr }}=0.06 \AA^{-1}$ for BSA at $150 \mathrm{mg} \mathrm{ml}{ }^{-1}$ without $\mathrm{NaCl}$ (Fig. 2a). This correlation peak can be related to a correlation length of $2 \pi / q_{\text {corr }}=10.5 \mathrm{~nm}$ in the solution. At higher BSA concentrations (250 and $500 \mathrm{mg} \mathrm{ml}^{-1}, c f$. Fig. 2c and e) the correlation length shifts to 9 and $7.4 \mathrm{~nm}$, respectively, pointing towards a pronounced crowding effect due to the increased protein concentration. The addition of $\mathrm{NaCl}$ flattens the correlation peak of the $150 \mathrm{mg} \mathrm{ml}^{-1}$ sample (see Fig. 2b). This effect is due to a NaCl-mediated charge screening and, thus, a decrease in electrostatic repulsion between BSA molecules. In the samples containing $250 \mathrm{mg} \mathrm{ml}^{-1}$ and $500 \mathrm{mg} \mathrm{ml}^{-1}$ BSA, the effect of $\mathrm{NaCl}$ on the correlation peak is less pronounced (Fig. $2 \mathrm{~d}$ and $\mathrm{f}$ ). We attribute this finding to the fact that at these high concentrations, the distances between BSA molecules are small, and caging effects on the structure are expected. ${ }^{57}$ Thus, the introduction of charge screening via $\mathrm{NaCl}$ leads to a less pronounced variation of proteinprotein distances than in the case of the $150 \mathrm{mg} \mathrm{ml}^{-1}$ sample.

Increasing the temperature to $311 \mathrm{~K}$ has little or no effect on the scattering profiles, outlining no significant structural effects in this temperature range. At $335 \mathrm{~K}$, all scattering profiles show an upturn in the low- $q$ region. While the low- $q$ upturn signifies emerging attraction and aggregation in the system, the correlation peak at around $0.06 \AA^{-1}$ is still visible. The latter feature suggests that the protein structure remains in rather compact domains with steric and electrostatic repulsion, but experiences additional attraction due to partial unfolding, as seen in the increase of $I(q \rightarrow 0)$. Interestingly, no significant changes in $I(q)$ are observed for $150 \mathrm{mg} \mathrm{ml}^{-1}$ at $335 \mathrm{~K}$, which might be due to the larger inter-protein distance rendering a possible short-ranged attraction less efficient.

The curves obtained upon further temperature increase to $T=348 \mathrm{~K}$ and also after cooling back to $295 \mathrm{~K}$ retain a pronounced low- $q$ upturn as a clear signature of cross-linking and network formation. As a general trend with increasing protein concentration, we observe a steepening of the low- $q$ upturn.

Interestingly, despite the strong attraction due to unfolding, a weaker correlation peak remains present for $500 \mathrm{mg} \mathrm{ml}^{-1}$ and $250 \mathrm{mg} \mathrm{ml}^{-1}$ even at high temperatures and upon cooling down, while the peak becomes insignificant for $150 \mathrm{mg} \mathrm{ml}$ at high temperatures and upon cooling. In addition, no strong effect of $\mathrm{NaCl}$ on the structures formed is observed at the higher protein concentrations (250 and $500 \mathrm{mg} \mathrm{ml}^{-1}$ ), while a significant structural difference exists for the aggregated network structures formed at $150 \mathrm{mg} \mathrm{ml}^{-1}$, as is evident for the clearly different slope at low $q$.

A possible structural interpretation is that, while the protein molecules remain in globular domains within an interconnected network formed upon denaturation, heat-induced partial unfolding induces a strong intermolecular attraction between the protein molecules located in these globular domains. This attraction is reflected in an overall aggregation behavior. Thus, we tentatively explain the different responses to $\mathrm{NaCl}$ observed for low and high
BSA concentrations as follows: at high BSA concentrations, the crowding effects are strong, meaning that the globular domains present even in the denatured network induce some weak order due to steric repulsion. On the contrary, the aggregated network structures formed at lower protein concentration experience less steric effects. They are therefore more open and disordered, and can thus respond more sensitively to effects of NaCl-induced charge screening.

Notably, the sample containing $150 \mathrm{mg} \mathrm{ml}^{-1}$ BSA without $\mathrm{NaCl}$ still changes significantly upon cooling down. This effect is no longer visible in the sample containing $150 \mathrm{mg} \mathrm{ml}^{-1} \mathrm{BSA}$ and $150 \mathrm{mM} \mathrm{NaCl}$ as well as in all other samples with higher BSA concentrations. We tentatively attribute this observation to a potential aging or slow kinetic effect of the denatured state. As an alternative explanation, we note that this post-denaturation effect could also be traced back to an equilibration process of re-compaction of local domains in the protein upon cooling, as suggested based on dynamical data. ${ }^{38}$

In order to quantify the effects of temperature on the samples, we compare the low $q$ regions up to $q=0.02 \AA^{-1}$ with power law curves $\left(I(q) \propto q^{-D}\right) .{ }^{44-46}$ The values of $D$ are indicated in the respective plots in Fig. 2 . We remark that the exponent is usually interpreted as a fractal dimension for these kinds of networkforming systems. ${ }^{44-46}$ In this interpretation, we observe a transition from more open structures with $D \approx 2$ corresponding to random-chain-like configurations at lower protein concentrations to steeper signatures for $500 \mathrm{mg} \mathrm{ml}{ }^{-1}$, which signify more compact objects. We remark that the $150 \mathrm{mg} \mathrm{ml}^{-1}$ BSA solution without $\mathrm{NaCl}$ cannot be interpreted as a fractal object, but rather appears to be an elongated aggregated object with loose and disordered internal structure. We stress that all samples decay consistent with the Porod scaling of $q^{-4}$ at larger $q$ (cf. Fig. 2), which evidences overall compact structures of the protein building blocks even in the aggregated networks.

\subsection{QENS: dynamical evolution during denaturation}

Having investigated the static properties of the samples via SANS, we employed QENS measurements in order to assess the changes in BSA dynamics throughout the process of thermal denaturation. While protein denaturation has been studied in detail using spectroscopic and calorimetric techniques, the understanding of dynamics inside a denatured protein network is very limited. In particular, it is unclear to which extent intramolecular motions are hindered by cross-linked and neighboring protein chains already on small time and length scales, or simply slowed down due to increased hydrodynamic friction in the local neighborhood of the protein molecules. This information would be essential to quantitatively describe macroscopic properties of protein networks such as their rheology, in the spirit of hierarchical models of protein dynamics.

We focus our discussion on four systems - lower-concentration samples containing $150 \mathrm{mg} \mathrm{ml}^{-1}$ BSA with and without $150 \mathrm{mM}$ $\mathrm{NaCl}$ and high-concentration samples containing $500 \mathrm{mg} \mathrm{ml}$ BSA with and without $150 \mathrm{mM} \mathrm{NaCl}$.

From the fits shown in Fig. 1 we obtain the mean-squared displacement values $\left\langle u^{2}\right\rangle(T)$ for the samples containing 150 and 
$500 \mathrm{mg} \mathrm{ml}^{-1}$ BSA in the absence and presence of $150 \mathrm{mM} \mathrm{NaCl}$, respectively. This heuristic definition provides an approach to compare different data sets without detailed knowledge of the different contributions to the elastic signal measured.

The systems studied show similar trends of $\left\langle u^{2}\right\rangle$ with $T$. With increasing $T,\left\langle u^{2}\right\rangle$ increases linearly until $T \approx 330 \mathrm{~K}$, reflecting an increase in global protein motion due to the higher thermal energy of the system. At $T>330 \mathrm{~K}$, a decrease of the $\left\langle u^{2}\right\rangle$ values is observed until $T \approx 365 \mathrm{~K}$. The intuitive interpretation backed up by the SANS data is that proteins partially unfold in this temperature regime, and subsequently form a network-like structure in which protein mobility is strongly decreased. Cooling the system back to $295 \mathrm{~K}$ does not restore the initial mobility as is seen in the low $\left\langle u^{2}\right\rangle$ values in all cases, thus pointing towards an irreversible BSA denaturation at high $T$.

Comparing the values of $\left\langle u^{2}\right\rangle$ of both systems, it is well visible that the overall values are lower in the system containing $500 \mathrm{mg} \mathrm{ml}{ }^{-1}$ BSA than in the one with $150 \mathrm{mg} \mathrm{ml}^{-1}$. This observation is expected given that a higher degree of crowding in the high-concentration sample implies a stronger sterical hindrance especially of translational diffusion of the BSA molecules. ${ }^{57-59}$ This is consistent with the SANS data shown in the previous section where the crowding is reflected in smaller overall inter-particle distances as derived from the respective correlation peaks.

Only small effects of $\mathrm{NaCl}$ on the overall temperaturedependent behavior of $\left\langle u^{2}\right\rangle$ are observed before denaturation (Fig. 3 top). However, once denaturation has taken place, $\mathrm{NaCl}$ causes a smaller $\left\langle u^{2}\right\rangle$ both at high temperatures and during cooling. Stronger differences between the samples with and without $\mathrm{NaCl}$ are visible at high temperatures during heating (Fig. 3) (a) as well as during the cooling process in the case of the lower BSA concentration $\left(150 \mathrm{mg} \mathrm{ml}^{-1}\right)$. This observation reflects the SANS results discussed in the previous section - the higher the protein concentration, the less pronounced the effect of charge screening by $\mathrm{NaCl}$, which is consistent with a previous finding that strong steric interactions render electrostatics less effective in BSA solutions. ${ }^{56}$ We note that, in the presence of $\mathrm{NaCl}$, the $\left\langle u^{2}\right\rangle$ observed upon cooling have similar values at both 150 and $500 \mathrm{mg} \mathrm{ml}^{-1} \mathrm{BSA}$, indicating that the effect of $\mathrm{NaCl}$ might play a more important role than the protein concentration once denaturation has taken place.

From a theoretical perspective, the apparent mean-squared displacement $\left\langle u^{2}\right\rangle$ is given by the real time-dependent meansquared displacement $\left\langle\Delta r^{2}\right\rangle(t)$ averaged over the time resolution of the neutron spectrometer $\mathscr{R}(t)^{51,53,60}$

$$
\left\langle u^{2}\right\rangle=\int \mathrm{d} t \mathscr{R}(t)\left\langle\Delta r^{2}\right\rangle(t)
$$

Thus, $\left\langle u^{2}\right\rangle$ can, without further information, neither be interpreted as a pure confinement effect nor as a purely dynamical quantity. Instead, it is a measure of the distance that an average hydrogen in the protein has moved within the instrumental time window, which, in other words, is the dynamical confinement in the system.

In order to directly obtain information on the underlying dynamics, we therefore used the intensity ratio $A(q, 1.3 \mu \mathrm{eV})$ between IFWS and EFWS as shown in Fig. 3(c-f) for two $q$ values. A larger ratio implies a broader $S(q, \omega)$, and thus faster dynamics. Overall, we obtain behavior qualitatively similar to that of $\left\langle u^{2}\right\rangle$ : the ratio increases with temperature until denaturation sets in. After forming a network of denatured proteins, and cooling back, the values of the intensity ratio remain significantly smaller than those observed during heating, indicating, as expected, slower overall dynamics in the crosslinked network than in the native solution state.

For $500 \mathrm{mg} \mathrm{ml}^{-1} \mathrm{BSA}$, the effect of salt on the intensity ratio is almost negligible, while salt seems to induce slower dynamics after denaturation in $150 \mathrm{mg} \mathrm{ml}^{-1}$ BSA. This behavior might be linked to the more open structures formed for $150 \mathrm{mg} \mathrm{ml}$ compared to the more compact network structures for $500 \mathrm{mg} \mathrm{ml}^{-1}$ (cf. Fig. 2). Thus, electrostatic repulsion between protein molecules might lead to more motional freedom and hence faster local dynamics for $150 \mathrm{mg} \mathrm{ml}^{-1}$ compared to $500 \mathrm{mg} \mathrm{ml}^{-1}$.

The good agreement between $\left\langle u^{2}\right\rangle$ and $A(q, 1.3 \mu \mathrm{eV})$ indicates that dynamics are the dominating factor in $\left\langle u^{2}\right\rangle$ for these samples. This observation is reasonable, as translational diffusion and local fluctuations in the assembled networks are not expected to show strong confinement effects on nanosecond time scales, and might thus dominate the signatures. In this context, the weaker effect of $\mathrm{NaCl}$ on dynamics than on dynamical confinement for $500 \mathrm{mg} \mathrm{ml}^{-1}$ BSA could indicate a stronger confinement effect in dense protein networks, which is expected intuitively.

We used a generic two-state function to quantify the overall characteristics of all heating profiles ${ }^{22}$ (solid lines in Fig. 3):

$$
\begin{gathered}
f(T)=\left(a_{1}+b_{1} T\right)[1-\Theta(T)]+\left(a_{2}+b_{2} T\right) \Theta(T) \\
\Theta(T)=\frac{1}{1+\mathrm{e}^{\frac{T-T_{0}}{\Delta T}}}
\end{gathered}
$$

Here, $\Theta(T)$ is a smeared step function with the central temperature $T_{0}$ and the transition range $\Delta T . a_{1,2}$ and $b_{1,2}$ denote the intercept and slope of the low- $T$ and high- $T$ state. As an additional parameter, we extracted an estimation for the denaturation temperature as $T_{\mathrm{d}}=T_{0}-\alpha \Delta T$, at which a first step, i.e., a partial unfolding of the protein, induces the aggregation process. This estimation is based on the consideration that the onset of denaturation is given once a significant deviation from the initial linear behavior is observed. Choosing $\alpha=2.5$, we obtain $\Theta\left(T_{\mathrm{d}}\right)=0.075$ as a reasonable threshold for a significant change. We remark that other choices of $\alpha$ yield qualitatively similar trends.

BSA solutions with $150 \mathrm{mg} \mathrm{ml}^{-1}$ without salt (Fig. 3(a)) represent an exception in the fitting, as limited statistics imply that a constant profile is sufficient to represent the data. This flat profile is attributed to the comparably fast dynamics, which implies that the elastic scattering intensities have decreased to close to background levels on the $q$ range used for fitting.

The cooling profiles were fitted with a polynomial

$$
f(T)=\left(c+d T+e T^{2}\right)
$$



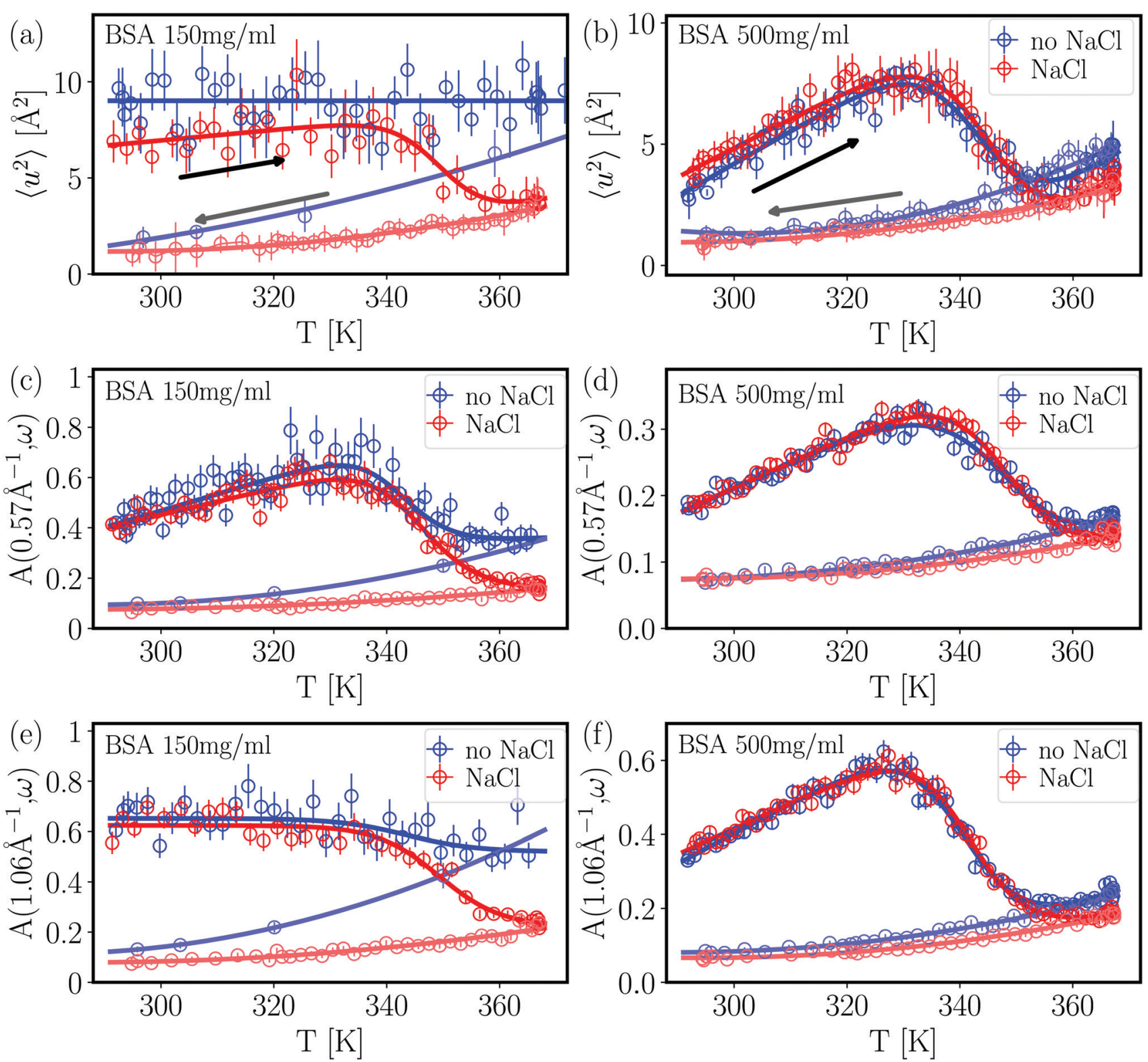

Fig. 3 Dynamical information from fixed window scans for $150 \mathrm{mg} \mathrm{ml}^{-1} \mathrm{BSA}$ (left column) and $500 \mathrm{mg} \mathrm{ml}^{-1} \mathrm{BSA}$ (right column). The arrows in plots (a) and (b) indicate the respective heating (right-pointing arrow) and cooling (left-pointing arrow) ramps performed during the experiments. Apart from the flat heating signature of BSA $150 \mathrm{mg} \mathrm{ml}^{-1}$ without $\mathrm{NaCl}$, all heating profiles are fitted with a two-state function with a smeared step function (eqn (6)). (a) and (b) Apparent mean-squared displacement $\left\langle u^{2}\right\rangle$ from EFWS. (c)-(f) Apparent intensity ratio $A(q, \omega=1.3 \mu$ eV) between IFWS and EFWS for two $q$ values. Data sets in (a) (BSA $150 \mathrm{mg} \mathrm{m}^{-1}$ without $\mathrm{NaCl}$ ) and (e) are binned by two data points for better visibility. All results show a decrease of dynamics upon the denaturation transition. Samples with $\mathrm{NaCl}$ show comparable dynamics before denaturation, and slower dynamics after denaturation. In general, results on dynamical confinement $\left\langle u^{2}\right\rangle$ from EFWS and dynamics from the ratio $A(q, \omega)$ of IFWS and EFWS show comparable trends, suggesting that the changes in $\left\langle u^{2}\right\rangle$ in these liquid samples are mainly driven by varying dynamics, and not by an evolution of the confinement.

with the parameters $c, d, e$. All fit parameters are summarized in Table 1.

As a first observation, the denaturation temperature $T_{\mathrm{d}}$ is comparably constant within error bars in all samples for both $\left\langle u^{2}\right\rangle$ and the ratios $A(q, \omega=1.3 \mu \mathrm{eV})$, except for BSA $150 \mathrm{mg} \mathrm{ml}^{-1}$ without NaCl. When comparing the values for $T_{\mathrm{d}}$ with and without $\mathrm{NaCl}$, an overall weak trend for lower $T_{\mathrm{d}}$ without salt may be present, suggesting a weak stabilizing effect of salt. This is consistent with results by Yamasaki and Yano ${ }^{20}$ who reported a NaCl-mediated stabilization of BSA against denaturation at neutral and alkaline $\mathrm{pH}$ values. In the present study, the salt effect on the dynamic behavior is, again, most pronounced for BSA solutions with $150 \mathrm{mg} \mathrm{ml}^{-1}$, which might indicate that an interplay of electrostatics and crowding plays a role for protein stability. The central temperature $T_{0}$ shows more fluctuations than $T_{\mathrm{d}}$. This is reasonable, since $T_{\mathrm{d}}$ is a purely thermodynamically determined quantity, while $T_{0}$ is additionally affected by kinetic contributions.

The observed dynamical signature of thermal denaturation contributes to a consistent picture from multiple techniques. 
Table 1 Fit parameters of FWS and IFWS/EFWS ratios shown in Fig. 3. The energy offset is $\omega_{1}=1.3 \mu \mathrm{eV}$. The $q$ values are $q_{1}=0.57 \AA^{-1}$ and $q_{2}=1.06 \AA^{-1}$

\begin{tabular}{|c|c|c|c|c|c|c|c|c|c|c|}
\hline Profile & \multicolumn{6}{|l|}{ Heating ramp } & \multicolumn{3}{|c|}{ Cooling ramp } & $T_{\mathrm{d}}^{b}[\mathrm{~K}]$ \\
\hline \multicolumn{11}{|c|}{ BSA $500 \mathrm{mg} \mathrm{ml}^{-1} \mathrm{NaCl} 0 \mathrm{M}$} \\
\hline$A\left(q_{1}, \omega_{1}\right)$ & $-2.16 \pm 1.04$ & $6.27 \pm 2.81$ & $-0.96 \pm 0.07$ & $3.91 \pm 0.24$ & $349.3 \pm 2.2$ & $6.82 \pm 0.77$ & $1.10 \pm 0.22$ & $-7.27 \pm 1.33$ & $12.8 \pm 2.0$ & $332.3 \pm 2.5$ \\
\hline$A\left(q_{2}, \omega_{1}\right)$ & $-1.94 \pm 0.45$ & $5.94 \pm 1.23$ & $-2.05 \pm 0.14$ & $8.17 \pm 0.45$ & $340.2 \pm 0.6$ & $5.49 \pm 0.45$ & $2.20 \pm 0.26$ & $-14.6 \pm 1.62$ & $25.3 \pm 2.5$ & $326.5 \pm 0.9$ \\
\hline \multicolumn{11}{|c|}{ BSA $500 \mathrm{mg} \mathrm{ml}^{-1} \mathrm{NaCl} 0.15 \mathrm{M}$} \\
\hline$A\left(q_{2}, \omega_{1}\right)$ & $-0.91 \pm 0.37$ & $296 \pm 102$ & $-1.66 \pm 0.12$ & $6.93 \pm 0.40$ & $341.1 \pm 0.5$ & $5.13 \pm 0.40$ & $1.94 \pm 0.18$ & $-12.7 \pm 1.1$ & $21.6 \pm 1.7$ & $328.3 \pm 0.8$ \\
\hline \multicolumn{11}{|c|}{ BSA $150 \mathrm{mg} \mathrm{ml}^{-1} \mathrm{NaCl} 0 \mathrm{M}$} \\
\hline$\left\langle u^{2}\right\rangle$ & $9.02 \pm 0.15$ & $0.00^{a}$ & $0.00^{a}$ & $0.00^{a}$ & $-\infty^{a}$ & $1.00^{a}$ & $18.7 \pm 53.7$ & $-160 \pm 313$ & $348 \pm 451$ & $0.00^{a}$ \\
\hline$A\left(q_{1}, \omega_{1}\right)$ & $-0.07 \pm 1.85$ & $1.18 \pm 5.09$ & $-1.54 \pm 0.25$ & $6.68 \pm 0.84$ & $342.1 \pm 2.5$ & $4.41 \pm 1.55$ & $3.47 \pm 2.51$ & $-23.5 \pm 15.8$ & $40.7 \pm 24.8$ & $331.1 \pm 3.5$ \\
\hline$A\left(q_{2}, \omega_{1}\right)$ & $0.50 \pm 0.06$ & $0.00^{a}$ & $0.65 \pm 0.02$ & $0.00^{a}$ & $342.8 \pm 9.2$ & $7.72 \pm 7.65$ & $5.00 \pm 3.86$ & $-35.0 \pm 24.5$ & $62.6 \pm 38.8$ & $325.9 \pm 12.2$ \\
\hline
\end{tabular}

First, starting around $330 \mathrm{~K}$, both $\mathrm{DSC}^{23}$ and our dynamical results show first signatures of denaturation, which might be related to a first conformational transition also observed via peak shifts in FTIR, which was attributed to an increased crevice between protein domains. ${ }^{21}$ At higher temperatures above $345 \mathrm{~K}$, the secondary structure is affected, as is visible by FTIR and CD spectroscopy, involving irreversible melting of $\alpha$ helices, ${ }^{21,23}$ which in our dynamical signatures leads to a stronger cross-linking. We remark that our SANS data together with earlier data from QENS $^{38}$ suggest that compact domains persist, but with a potentially altered secondary structure.

In powder samples, $\left\langle u^{2}\right\rangle$ is often used to define an effective force constant $k=\mathrm{d}\left\langle u^{2}\right\rangle / \mathrm{d} T$. While this approach would also be tempting for the cooling ramps, the clear polynomial signature implies that the dynamics are not governed by a universal force constant, but rather by an apparent force constant changing with temperature. This observation might be due to the fact that, upon cooling, the local levels of dynamics return to their initial values in the native structure, ${ }^{38}$ which suggests a local compaction of the protein structure. This compaction, in line with the overall fractal-like structure, could be a reason for a changing force constant. A second reason is that the polynomial signature is obtained for both $\left\langle u^{2}\right\rangle$ and the ratios $A(q, \omega)$. This suggests that the signature observed might be caused by the dynamical response within a network, and not by the confinement, which is the reasoning behind the effective force constant.

\subsection{Remarks on the methodology}

On a practical note, we stress that differences in the absolute MSD values $\left\langle u^{2}\right\rangle$ determined by EFWS depend on the $q$ range measured in the experiments. In addition, contrary to full QENS spectra, the values for $\left\langle u^{2}\right\rangle$ critically depend on a precise calibration. Finally, the extraction via the Gaussian approximation or also the heuristic polynomial approximation in eqn (2) is valid only for sufficiently small $q$, which turns out to be less problematic for powder samples than for proteins in solution. In this context, the use of the combined analysis of IFWS and EFWS is a way to obtain an independent set of information which can be successfully used to check and validate the overall interpretation. We remark that the ratio even seems to be statistically more robust than $\left\langle u^{2}\right\rangle$ for less concentrated samples with comparably fast diffusion, as seen for the case of the BSA solution with $150 \mathrm{mg} \mathrm{ml}^{-1}$ and without $\mathrm{NaCl}$.

The combined analysis of the elastic and inelastic fixed window data allows to access additional information on the evolution of (sub)nanosecond dynamics in the sample with a very good kinetic resolution on the order of a few minutes, contrary to full QENS spectra which require several hours. This option is very relevant to study the time-dependence of denaturation, and can be applied to other topics such as assembly and crystallization. ${ }^{52}$ In particular, the combination with conventional analysis of the EFWS allows to distinguish between effects of geometrical confinement and dynamics on nanosecond motions characterized by $\left\langle u^{2}\right\rangle$. While we observed a dominance of dynamics for liquid samples, it is interesting to explore to which extent $\left\langle u^{2}\right\rangle$, e.g. in powder samples, is governed by dynamics or confinement. Our analysis approach opens opportunities to study dynamical landscapes in more microscopic detail.

\section{Conclusions}

In this study, we applied SANS and QENS to study static and dynamic aspects of thermal BSA denaturation and the way it is influenced by the addition of NaCl. Using SANS, we show that at high BSA concentrations, the screening effect of $\mathrm{NaCl}$ is less efficient than at low concentrations and we attribute this observation to strong steric interactions. Upon denaturation, we observe a powerlaw-like upturn at low $q$ that indicates the formation of a fractal network, which persists also after cooling back to room temperature.

Using fixed window scans (FWS) of QENS, we characterize the evolution of dynamical confinement and dynamics during 
temperature ramps through the denaturation transition. All signatures indicate that a significant fraction of mobility is lost upon denaturation. Comparing the dynamical signal from ratios of inelastic and elastic FWS with the dynamical confinement obtained from the apparent mean-squared displacement, we suggest that the observed signatures of denaturation on nanosecond time scales are dominated by temperature-induced changes of the dynamics, whereas changes of the confinement only contribute weak effects. Thus, crosslinking does not seem to induce confinement of the motions observed on nanosecond time scales. This is a relevant finding for future quantitative modeling of, e.g., rheological properties of protein networks. Our framework combining different scattering methods presents a promising tool for a detailed study of structural and dynamical evolution during assembly processes, opening opportunities for fundamental understanding of processes in e.g. food and life sciences.

\section{Data accessibility}

The SANS and QENS data are permanently curated by the ILL and accessible via the DOIs 10.5291/ILL-DATA.9-13-637, ${ }^{61} 10.5291 /$ ILL-DATA.8-04-752, ${ }^{62} 10.5291 /$ ILL-DATA.9-13-672 ${ }^{63}$ and 10.5291/ILLDATA.8-04-759. ${ }^{64}$

\section{Conflicts of interest}

There are no conflicts to declare.

\section{Acknowledgements}

The authors thank the ILL for beamtime allocation on beamlines D11 and IN16B. Financial support by the DFG (DFG 316738961) and the ANR (ANR-16-CE92-0009, ImmunoglobulinCrowding) as well as by the BMBF is gratefully acknowledged. C. B. acknowledges a PhD studentship cofunded by the ILL and University of Tübingen. O. M. acknowledges a PhD fellowship by the Studienstiftung des deutschen Volkes. L. B. acknowledges an ILL studentship to fund the research for her Diploma thesis carried out in the ILL spectroscopy group. F. R.-R. acknowledges funding from the Royal Physiographic Society of Lund. The authors thank the ILL-ESRF Partnership for Soft and Condensed Matter (PSCM, Grenoble) for sharing lab resources.

\section{References}

1 A. L. Lehninger, D. L. Nelson and M. M. Cox, Lehninger Principles of Biochemistry, W. H. Freeman, New York, 2005.

2 S. Timr, D. Madern and F. Sterpone, in Computational Approaches for Understanding Dynamical Systems: Protein Folding and Assembly, ed. B. Strodel and B. Barz, Progress in Molecular Biology and Translational Science, Academic Press, 2020, vol. 170, pp. 239-272.

3 J. Gunton, A. Shiryayev and D. Pagan, Protein Condensation: Kinetic Pathways to Crystallization and Disease, Cambridge University Press, 2007.
4 E. L. Opie, J. Exp. Med., 1962, 115, 597-608.

5 G. B. Benedek, Invest. Ophthalmol. Visual Sci., 1997, 38, 1911-1921.

6 C. Kato and D. H. Bartlett, Extremophiles, 1997, 1, 111-116.

7 D. J. Kushner, Adv. Appl. Microbiol., 1968, 10, 73-99.

8 A. Ventosa, J. J. Nieto and A. Oren, Microbiol. Mol. Biol. Rev., 1998, 62, 504-544.

9 P. Kanokratana, S. Chanapan, K. Pootanakit and L. Eurwilaichitr, J. Basic Microbiol., 2004, 44, 430-444.

10 J. Smiatek, J. Phys. Chem. B, 2014, 118, 771-782.

11 J. K. Lanyi, Bacteriol. Rev., 1974, 38, 272-290.

12 R. Jaenicke, Eur. J. Biochem., 1991, 202, 715-728.

13 R. Jaenicke and G. Böhm, Curr. Opin. Struct. Biol., 1998, 8, 738-748.

14 D. Madern, C. Ebel and G. Zaccai, Extremophiles, 2000, 4, 91-98.

15 R. Sterner and W. Liebl, Crit. Rev. Biochem. Mol. Biol., 2001, 36, 39-106.

16 A. E. I. Coskun, D. Sağlam, P. Venema, E. van der Linden and E. Scholten, Food Hydrocolloids, 2015, 45, 291-300.

17 S. Scheingraber, M. Rehm, C. Sehmisch and U. Finsterer, Anesthesiology, 1999, 90, 1265-1270.

18 D. S. Prough and A. Bidani, Anesthesiology, 1999, 90, 1247-1249.

19 C. Giancola, C. De Sena, D. Fessas, G. Graziano and G. Barone, Int. J. Biol. Macromol., 1997, 20, 193-204.

20 M. Yamasaki, H. Yano and K. Aoki, Int. J. Biol. Macromol., 1990, 12, 263-268.

21 K. Murayama and M. Tomida, Biochemistry, 2004, 43, 11526-11532.

22 M. Hennig, F. Roosen-Runge, F. Zhang, S. Zorn, M. W. A. Skoda, R. M. J. Jacobs, T. Seydel and F. Schreiber, Soft Matter, 2012, 8, 1628-1633.

23 J. I. Boye, I. Alli and A. A. Ismail, J. Agric. Food Chem., 1996, 44, 996-1004.

24 A. Tobitani and S. B. Ross-Murphy, Macromolecules, 1997, 30, 4845-4854.

25 A. Tobitani and S. B. Ross-Murphy, Macromolecules, 1997, 30, 4855-4862.

26 J. Lefebvre, D. Renard and A. C. Sanchez-Gimeno, Rheol. Acta, 1998, 37, 345-357.

27 G. Barone, C. Giancola and A. Verdoliva, Thermochim. Acta, 1992, 199, 197-205.

28 V. Vetri, F. Librizzi, M. Leone and V. Militello, Eur. Biophys. J., 2007, 36, 717-725.

29 V. Militello, V. Vetri and M. Leone, Biophys. Chem., 2003, 105, 133-141.

30 V. Militello, C. Casarino, A. Emanuele, A. Giostra, F. Pullara and M. Leone, Biophys. Chem., 2004, 107, 175-187.

31 M. Bhattacharya, N. Jain and S. Mukhopadhyay, J. Phys. Chem. B, 2011, 115, 4195-4205.

32 M. Sogami and J. F. Foster, Biochemistry, 1968, 7, 2172-2182. 33 A. Del Giudice, C. Dicko, L. Galantini and N. V. Pavel, J. Phys. Chem. B, 2017, 121, 4388-4399.

34 A. Del Giudice, C. Leggio, N. Balasco, L. Galantini and N. V. Pavel, J. Phys. Chem. B, 2014, 118, 10043-10051.

35 M. Grimaldo, F. Roosen-Runge, F. Zhang, F. Schreiber and T. Seydel, Q. Rev. Biophys., 2019, 52, 1-63. 
36 F. Ameseder, A. Radulescu, M. Khaneft, W. Lohstroh and A. M. Stadler, Phys. Chem. Chem. Phys., 2018, 20, 5128-5139.

37 F. Ameseder, A. Radulescu, O. Holderer, P. Falus, D. Richter and A. M. Stadler, J. Phys. Chem. Lett., 2018, 9, 2469-2473.

38 M. Grimaldo, F. Roosen-Runge, M. Hennig, F. Zanini, F. Zhang, N. Jalarvo, M. Zamponi, F. Schreiber and T. Seydel, Phys. Chem. Chem. Phys., 2015, 17, 4645-4655.

39 J. S. Pedersen, Adv. Colloid Interface Sci., 1997, 70, 171-210.

40 P. Lindner and T. Zemb, Neutrons, X-rays, and Light: Scattering Methods Applied to Soft Condensed Matter, Elsevier, NorthHolland, 2002.

41 P. Debye, Ann. Phys., 1915, 351, 809-823.

42 J.-P. Hansen and I. R. McDonald, Theory of simple liquids, Academic Press, Amsterdam, 2006.

43 L. A. Feigin and D. I. Svergun, Structure Analysis by Small-Angle X-ray and Neutron Scattering, Plenum Press, New York, 1987.

44 A. Guinier and G. Fournet, Small-angle scattering of X-rays, Wiley, New York, 1955, vol. 14.

45 J. Teixeira, J. Appl. Crystallogr., 1988, 21, 781-785.

46 E. Mircea Anitas, Nanomaterials, 2019, 9, 648.

47 K. Lieutenant, P. Lindner and R. Gähler, J. Appl. Crystallogr., 2007, 40, 1056-1063.

48 M. Könnecke, F. A. Akeroyd, H. J. Bernstein, A. S. Brewster, S. I. Campbell, B. Clausen, S. Cottrell, J. U. Hoffmann, P. R. Jemian and D. Männicke, et al., J. Appl. Crystallogr., 2015, 48, 301-305.

49 B. Frick, J. Combet and L. van Eijck, Nucl. Instrum. Methods Phys. Res., Sect. A, 2012, 669, 7-13.

50 C. Beck, M. Grimaldo, F. Roosen-Runge, R. Maier, O. Matsarskaia, M. Braun, B. Sohmen, O. Czakkel,
R. Schweins, F. Zhang, T. Seydel and F. Schreiber, Cryst. Growth Des., 2019, 19, 7036-7045.

51 F. Roosen-Runge and T. Seydel, EPJ Web Conf., 2015, 83, 02015.

52 G. Zaccai, Science, 2000, 288, 1604-1607.

53 R. Zorn, Nucl. Instrum. Methods Phys. Res., Sect. A, 2009, 603, 439-445.

54 J. Peters and G. R. Kneller, J. Chem. Phys., 2013, 139, 135102. 55 D. Zeller, M. T. F. Telling, M. Zamponi, V. García Sakai and J. Peters, J. Chem. Phys., 2018, 149, 234908.

56 F. Roosen-Runge, M. Hennig, T. Seydel, F. Zhang, M. W. Skoda, S. Zorn, R. M. Jacobs, M. Maccarini, P. Fouquet and F. Schreiber, Biochim. Biophys. Acta, Proteins Proteomics, 2010, 1804, 68-75.

57 M. Grimaldo, F. Roosen-Runge, F. Zhang, T. Seydel and F. Schreiber, J. Phys. Chem. B, 2014, 118, 7203-7209.

58 F. Roosen-Runge, M. Hennig, F. Zhang, R. M. J. Jacobs, M. Sztucki, H. Schober, T. Seydel and F. Schreiber, Proc. Natl. Acad. Sci. U. S. A., 2011, 108, 11815-11820.

59 M. Grimaldo, H. Lopez, C. Beck, F. Roosen-Runge, M. Moulin, J. M. Devos, V. Laux, M. Härtlein, S. Da Vela, R. Schweins, A. Mariani, F. Zhang, J.-L. Barrat, M. Oettel, V. T. Forsyth, T. Seydel and F. Schreiber, J. Phys. Chem. Lett., 2019, 10, 1709-1715.

60 S. Magazu, F. Migliardo and A. Benedetto, J. Phys. Chem. B, 2010, 114, 9268-9274.

61 Institut Laue-Langevin, DOI: 10.5291/ILL-DATA.9-13-637. 62 Institut Laue-Langevin, DOI: 10.5291/ILL-DATA.8-04-752. 63 Institut Laue-Langevin, DOI: 10.5291/ILL-DATA.9-13-672. 64 Institut Laue-Langevin, DOI: 10.5291/ILL-DATA.8-04-759. 